\title{
Dietary supplementation with $n-3$ PUFA does not promote weight loss when combined with a very-low-energy diet
}

\author{
Irene A. Munro and Manohar L. Garg* \\ Nutraceuticals Research Group, School of Biomedical Sciences and Pharmacy, 305C Medical Sciences Building, \\ The University of Newcastle, Callaghan, NSW 2308, Australia
}

(Submitted 14 September 2011 - Final revision received 9 November 2011 - Accepted 11 November 2011 - First published online 3 January 2012)

\begin{abstract}
Obesity is associated with elevated levels of inflammation and metabolic abnormalities which are linked to CVD. The aim of the present study was to investigate whether long-chain $n$-3 PUFA (LCn-3PUFA), combined with a very-low-energy diet (VLED), facilitated weight loss and weight maintenance, and improvements in blood lipids and inflammatory mediators. This was a double-blind, randomised, controlled trial with two parallel groups. For 14 weeks, one group consumed $6 \times 1 \mathrm{~g}$ capsules/d of monounsaturated oil (placebo group, PB), and the other group consumed $6 \times 1 \mathrm{~g}$ capsules/d of LC $n$-3PUFA (fish oil group, FO), each comprising 70 mg EPA and 270 mg DHA. Both groups were on VLED for 4 weeks ( $n 14 \mathrm{~PB}, n 18 \mathrm{FO}$ ), which was then followed by 10 weeks of weight maintenance ( $n 12 \mathrm{~PB}, n 17 \mathrm{FO}$ ). Fasting blood samples, anthropometric measurements and $3 \mathrm{~d}$ food diaries were collected at baseline, at 4 and 14 weeks. A greater-than2 -fold increase occurred in plasma levels of EPA and DHA in the FO group $(P<0 \cdot 001)$. At 4 weeks, the mean weight loss was -6.54 (SD 2.08) $\mathrm{kg}(-6.9 \%)$ for $\mathrm{PB}$ and -6.87 (SD 1.83) $\mathrm{kg}(-7.7 \%)$ for FO. At week 14, after the maintenance phase, there was a further mean decrease in weight, -1.57 (SD 3.7$) \mathrm{kg}(1.85 \%)$ for PB and -1.69 (sD 2.32) kg (-1.9\%) for FO. Both groups experienced improved metabolic profiles and there was a significant reduction in fat mass for the FO group at week 14 but not for PB. However, it would appear that supplementation with LC $n$-3PUFA had no significant effect on weight loss or weight maintenance over the 14 weeks.
\end{abstract}

\section{Key words: Long-chain n-3 PUFA: Weight loss: Weight maintenance: Very-low-energy diets}

Obesity is a progressive and relapsing chronic disorder that is associated with increased risk of morbidity and mortality including the development of insulin resistance, type 2 diabetes, hypertension, dyslipidaemia ${ }^{(1)}$ and cholesterol abnormalities $^{(2)}$. It is also associated with low-grade systemic inflammation through the expression of proinflammatory mediators such as TNF- $\alpha$, IL- ${ }^{(3)}$, C-reactive protein (CRP) ${ }^{(4)}$ and leptin ${ }^{(5)}$, all of which have been linked to increased risk of $\mathrm{CVD}^{(6)}$. Adipose tissue also secretes the anti-inflammatory mediator, adiponectin, which has direct positive effects on vascular function ${ }^{(7)}$.

Intentional weight loss with a reduction of adipose tissue improves many of these medical complications with a decrease in circulating levels of proinflammatory biomarkers $^{(8,9)}$ and a reduction of metabolic abnormalities ${ }^{(10)}$. Dietary supplementation with long-chain n-3 PUFA (LCn-3PUFA) is also known to suppress the production of proinflammatory cytokines ${ }^{(11)}$ and increase the levels of HDL-cholesterol (HDL-C) ${ }^{(12,13)}$. LCn-3PUFA also lower TAG levels $^{(13,14)}$ and to a considerably lower level than weight loss does ${ }^{(15)}$. Animal studies have shown that supplementing the diet with LCn-3PUFA can reduce body weight and fat mass (FM) in mice ${ }^{(16,17)}$ and it is possible that, in humans, LCn-3PUFA might also reduce body fat ${ }^{(18)}$ and could have a similar effect on body weight.

There is a dose-dependent relationship with weight loss and improvements to health; and it has been suggested that a modest weight loss of 5-10\% of initial body weight can make a difference ${ }^{(19,20)}$. There is a possibility that LCn-3PUFA could facilitate this by increasing weight loss when taken in combination with a weight loss programme. The aim of this study was to investigate the effect of LCn-3PUFA on weight loss and short-term weight loss maintenance in obese adults by comparing two groups who followed the same very-lowenergy diet (VLED) using meal replacements (MR), with one group consuming fish oil capsules. We hypothesised that (i) LCn-3PUFA of marine origin may facilitate weight loss in combination with a weight loss programme using a VLED, and that (ii) continued supplementation with LCn-3PUFA may inhibit weight regain during a period of weight maintenance.

Abbreviations: CRP, C-reactive protein; FFM, fat-free mass; FM, fat mass; FO, fish oil; HDL-C, HDL-cholesterol; LC $n$-3PUFA, long-chain $n$-3 PUFA; LDL-C, LDL-cholesterol; PB, placebo; TC, total cholesterol; VLED, very-low-energy diet; WC, waist circumference.

*Corresponding author: Professor M. L. Garg, fax +61 24921 2028, email manohar.garg@newcastle.edu.au 
Furthermore, the combination of weight loss and LC n-3PUFA supplementation may have a concomitant positive effect on cardiovascular health determinants.

\section{Methods}

This was a randomised, double-blind, placebo-controlled intervention with two parallel groups. This study was conducted according to the guidelines laid down in the Declaration of Helsinki and all procedures were approved by the Human Research Ethics Committee of the University of Newcastle, Australia. Written informed consent was obtained from all participants before commencement of the study. The trial was registered with the Australian New Zealand Clinical Trials Registry (ACTRN12609000204246).

\section{Participants}

Both male and female participants, aged 18-60 years and with a BMI of between 30 and $40 \mathrm{~kg} / \mathrm{m}^{2}$, were recruited from the university campus and the general public in Newcastle, Australia to take part in a weight loss study. People with diagnosed diabetes mellitus, a chronic inflammatory condition, already following an energy-restricted diet, allergic to fish, taking fish oil capsules or consuming two or more oily fish meals per week were excluded from the study, as were women who were pregnant or lactating.

A power calculation estimated that sixteen participants per group would allow a detection of approximately $2 \%$ difference in weight loss between the two diet groups at the 0.05 level of significance, with a statistical power of $80 \%$ and assuming a standard deviation of $2 \%$. Allowing for a $20 \%$ dropout rate, it was estimated that twenty participants per group would be required.

\section{Study design}

Simple randomisation using computer-generated random numbers allocated participants to one of two parallel groups. Supplement containers were labelled with a code by an independent researcher before the trial commenced so that neither the principal researcher nor the participants could identify the contents of the supplements, and supplements contained peppermint to disguise identifiable flavours. For 4 weeks, one group consumed $6 \times 1 \mathrm{~g}$ capsules of placebo per day (placebo group, PB), containing Sunola oil (monounsaturated oil), and the other group consumed $6 \times 1 \mathrm{~g}$ capsules of fish oil per day (fish oil group, FO). Each fish oil capsule contained tuna oil comprising $360 \mathrm{mg}$ LC $n-3$ PUFA with $70 \mathrm{mg}$ EPA and $270 \mathrm{mg}$ DHA (ratio, 1:3.9). Both the tuna oil and the Sunola oil contained the antioxidant Coviox ${ }^{\circledR} \mathrm{T}-70,3000$ parts per million in the tuna oil and 600 parts per million in the Sunola oil. Two capsules were consumed with each meal (breakfast, lunch and dinner), providing $1.62 \mathrm{~g} \mathrm{DHA}$ and $0.42 \mathrm{~g} \mathrm{EPA} / \mathrm{d}$.

For the 4 weeks, participants followed a VLED of $3000 \mathrm{~kJ} / \mathrm{d}$. To encourage dietary compliance and to reduce differences between participants in the foods that they consumed, for the first 2 weeks participants received Optifast ${ }^{\circledR}$ bars and shakes to replace meals, according to the Intensive Phase of the Optifast Very Low Calorie Diet Program (Novartis, Australia), supplemented daily with raw and cooked vegetables and 2 litres of drinking-water. This $3000 \mathrm{~kJ} / \mathrm{d}$ diet is composed of $40 \%$ protein, $16 \%$ fat and $40 \%$ carbohydrate plus vitamins and minerals. At the same time, participants were given individual nutrition education and counselling, with the sessions focusing on the energy density of foods, understanding and using of food labels, appropriate portion sizes and the number of portions to be consumed daily from the different food groups. This information was used to build a healthy diet using the guidelines from the Australian Guide to Healthy Eating ${ }^{(21)}$, and applied during weeks 3 and 4, when the MR were gradually phased out and healthy energy-controlled meals were phased in. All participants attended weekly so that weight loss progress could be monitored and to receive the next supply of MR and capsules.

To address the potential for weight loss rebound after the weight loss phase, participants progressed to a weight stabilisation/maintenance phase. For 10 weeks, they continued to take the fish oil or placebo supplements as allocated in the weight loss phase, and continued to consume healthy meals with food choices and portion sizes as recommended by the Australian Guide to Healthy Eating. Participants now attended fortnightly to obtain their capsules, monitor their weight and discuss diet-related issues. An accredited practising dietitian was part of the research team to advise on healthcare.

\section{Dietary assessment}

Before commencing the weight loss programme, dietary intake was assessed with a $3 \mathrm{~d}$ food diary (two weekdays and one weekend day) to determine everyday nutrient and energy intake. Participants used the weights given on food products and handy measures of cups and spoons to record food intake which was analysed using the dietary software program, Foodworks ${ }^{\circledR}$ Professional 2009, version 6 (Xyris Software (Australia) Private Limited), and the mean values were calculated. In the final week of weight maintenance, participants again completed a $3 \mathrm{~d}$ food diary to measure changes in dietary intake.

\section{Anthropometric assessment}

On the first day of the weight loss trial, anthropometric measurements were taken in the morning after a $10 \mathrm{~h}$ overnight fast and no alcohol consumption, with participants dressed in light clothing and without shoes. Standing height was measured to the nearest $0 \cdot 1 \mathrm{~cm}$ using a stadiometer. Body weight was measured to the nearest $0 \cdot 1 \mathrm{~kg}$ using a calibrated balance beam scale (PCS Measurement). BMI was calculated in $\mathrm{kg} / \mathrm{m}^{2}$ from weight and height. Waist circumference (WC) was measured at the mid-point between the lowest rib and the top of the hipbone; the hip measurement was taken at the fullest point of the hip, as viewed from the side. The waist:hip ratio was calculated as waist girth $(\mathrm{cm})$ divided by the hip girth $(\mathrm{cm})$. FM, fat-free mass (FFM) and 
muscle mass were measured using single-frequency bioelectrical impedance (Maltron International) and (InBody 230; Biospace Company, Limited). Under identical conditions, these anthropometric measurements were repeated at the end of the weight loss and weight maintenance phases.

\section{Biochemical analyses}

Blood sample collection. Fasting blood samples were collected into tubes, EDTA, lithium heparin and sodium fluoride, by venepuncture at baseline, at the end of the weight loss phase and again at the end of weight maintenance. The samples were prepared by centrifuging (Heraeus Biofuge Stratos) for $10 \mathrm{~min}$ at $3000 \mathrm{~g}$ at $4^{\circ} \mathrm{C}$. Plasma samples from the EDTA tubes were collected and stored at $-80^{\circ} \mathrm{C}$ until further analysis. Plasma from the lithium heparin and sodium fluoride tubes was analysed for blood lipids and blood glucose, respectively, by Hunter Area Pathology Services.

\section{Plasma fatty acid analysis}

The plasma lipids were analysed for fatty acid composition using an acetyl chloride methylation procedure, a modification of the method of Lepage \& Roy ${ }^{(22)}$. Fatty acid methyl esters were quantified using a GC (Hewlett Packard 6890; Hewlett Packard) and identified by comparing peak retention times with the retention times of synthetic standards of known fatty acid composition (Nu Check Prep).

\section{Analysis of inflammatory markers}

ELISA kits (R\&D Systems) were used to analyse inflammatory markers. The assays used to determine plasma leptin and adiponectin levels employed the quantitative sandwich enzyme immunoassay technique with a detection limit of $15.6 \mathrm{pg} / \mathrm{ml}$ and $3.9 \mathrm{ng} / \mathrm{ml}$, respectively, and inter- and intra-assay CV of less than 5.5 and $7 \%$, respectively. High-sensitivity ELISA kits were used to determine levels of TNF- $\alpha$ (high-sensitivity TNF- $\alpha$ ) and IL-6 (high-sensitivity IL-6). Minimal detectable concentration of these kits was 0.106 and $0.039 \mathrm{pg} / \mathrm{ml}$, respectively, with intra- and inter-assay $\mathrm{CV}$ of $<9 \%$. Analysis of high-sensitivity CRP was performed using an immunoturbidimetric method (Hunter Area Pathology Services) with a minimal detection level of $0 \cdot 15 \mathrm{mg} / \mathrm{l}$.

\section{Statistical analyses}

ANOVA was used to test group mean differences; and withingroup changes from baseline were determined using pairedsamples $t$ tests. Pearson product-normal correlations $(r)$ were used to show relationships. Data are presented as mean values and standard deviations, with $P \leq 0.05$ indicating significance. All statistical analyses were carried out using SPSS software (version 17.0; SPSS, Inc.).

\section{Results}

A total of forty participants commenced the present study (twenty per group), but eight did not complete the study; two reacted to the supplements, one participant relocated to another city, one withdrew for personal reasons, one withdrew because of a broken ankle and three participants did not keep appointments for final measurements and blood collection. Hence, thirty-two participants completed the weight loss phase, eighteen in the FO group (fifteen females and three males) and fourteen in the PB group (eleven females and three males). Extreme outliers were

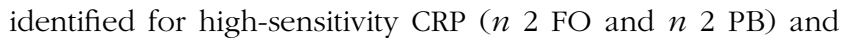
were excluded from the analysis of this biomarker. The thirty-two participants continued to the weight stabilisation/ maintenance phase, but only twenty-nine completed this phase; as three participants did not keep their appointments for final measurements and blood collection (one female $\mathrm{FO}$, and one male and one female PB). In the weight maintenance phase, other extreme outliers were identified and excluded ( $n 1 \mathrm{~PB}$ for glucose and $n 2$ FO for adiponectin).

\section{Diet}

Mean energy intake of the participants before commencing the study was 8273 (SD 1669) kJ/d for FO and 7846 (SD 2399) $\mathrm{kJ} / \mathrm{d}$ for PB; the differences were not significant. During weight loss, all participants followed a very similar $3000 \mathrm{~kJ} / \mathrm{d}$ MR diet for 4 weeks. The $3 \mathrm{~d}$ food diaries completed towards the end of the maintenance phase showed a mean energy intake of 5080 (SD 1294) kJ/d for FO and 6007 (SD 1758) kJ/d for PB. Again, the differences between the two groups were not significant.

The percentage fatty acid composition of plasma lipids at baseline showed no significant difference between the two groups. Supplementation with LC $n-3$ PUFA in FO significantly increased EPA $(\mathrm{C} 20: 5 n-3)(P<0.001)$ and DHA $(\mathrm{C} 22: 6 n-3)(P<0 \cdot 001)$ (Table 1$)$, and these increased levels

Table 1. Baseline values and changes for long-chain $n-3$ PUFA (LCn3-PUFA)

(Mean values and standard deviations)

\begin{tabular}{|c|c|c|c|c|c|}
\hline \multirow[b]{2}{*}{ LCn3-PUFA } & \multicolumn{2}{|c|}{ Baseline (\%) } & \multicolumn{2}{|c|}{4 weeks (\%) } & \multirow[b]{2}{*}{$P$} \\
\hline & Mean & SD & Mean & SD & \\
\hline \multicolumn{6}{|c|}{$(20: 5 n-3)$ EPA $(\%)$} \\
\hline $\mathrm{FO}^{*}$ & 1.02 & 0.30 & $2 \cdot 10$ & 0.45 & $<0.001$ \\
\hline $\mathrm{PB} \dagger$ & 0.88 & 0.26 & 0.78 & 0.37 & NS \\
\hline \multicolumn{6}{|c|}{$(22: 6 n-3)$ DHA (\%) } \\
\hline $\mathrm{FO}^{*}$ & 2.05 & 0.54 & 5.35 & 0.93 & $<0.001$ \\
\hline PB十 & 2.07 & 0.57 & 2.48 & 0.47 & NS \\
\hline \multicolumn{6}{|l|}{ Ratio $n-6 / n-3$} \\
\hline $\mathrm{FO}^{*}$ & 8.75 & $2 \cdot 00$ & 4.33 & 1.16 & $<0.001$ \\
\hline $\mathrm{PB} \dagger$ & 8.90 & 1.59 & 8.47 & 1.40 & NS \\
\hline \multicolumn{6}{|c|}{ Ratio $18: 1 / 18: 0$} \\
\hline $\mathrm{FO}^{*}$ & $2 \cdot 90$ & 0.51 & 3.02 & 0.44 & NS \\
\hline $\mathrm{PB} \dagger$ & $2 \cdot 89$ & 0.35 & 3.35 & 0.61 & $<0.01$ \\
\hline$\Delta$ & \multicolumn{2}{|c|}{0.01} & \multicolumn{2}{|c|}{0.33} & $<0.05$ \\
\hline
\end{tabular}


were significantly higher than those levels found in $\mathrm{PB}$ at the end of weight loss ( $P<0.001$ for both). Levels of EPA and DHA remained significantly higher for $\mathrm{FO}$ than for $\mathrm{PB}$ at the end of weight maintenance $(P<0.001$ for both).

\section{Anthropometric measurements}

Weight loss phase. At the commencement of the study, the baseline measurements for weight, BMI, FM, FFM, waist and hip were not significantly different between $\mathrm{PB}$ and FO (Table 2). At the end of 4 weeks of weight loss, there was a significant reduction in weight $-7.7 \%(-6.87 \mathrm{~kg}), \mathrm{FM}-15 \%$ $(-5.47 \mathrm{~kg})$ and FFM $-2.6 \%(-1.36 \mathrm{~kg})$ in FO, $(P<0.001$ for all). In $\mathrm{PB}$, there were also significant reductions in weight $-6.9 \%(-6.54 \mathrm{~kg})$ and $\mathrm{FM}-13.9 \%(-5.53 \mathrm{~kg})(P<0.001$ for both), and in FFM $-1.8 \%(-1.0 \mathrm{~kg})(P<0.02)$. For both FO and $\mathrm{PB}$, there was a significant reduction in $\mathrm{WC}-6.4 \%$ $(-6.53 \mathrm{~cm})$ and $-4.8 \%(-4.96 \mathrm{~cm})$, respectively; and also in hip measurement $-3.7 \%(-4.44 \mathrm{~cm})$ and $-3.5 \%(-4.23 \mathrm{~cm})$ for FO and PB, respectively ( $P<0.001$ for all) (Table 2 ). There was no significant difference in the changes between the two groups. Controlling for BMI using two-stage multiple regression confirmed this result. Correlations between changes in anthropometric measurements, weight loss and LCn-3PUFA were computed. There were positive, significant correlations between weight loss and reductions to BMI and FM $(P<0.001$ for $\mathrm{FO}$ and $\mathrm{PB})$, and between weight loss and reductions to FFM $(P<0.05$ FO and $P<0.001 \mathrm{~PB})$. There was also a positive, significant correlation between increased levels of EPA and a reduction in FM $(P<0 \cdot 02)$ in FO.

Weight maintenance phase. The number of participants completing this phase reduced by two in PB to $n 12$, and by one in FO to $n$ 17. Table 2 shows the reductions in anthropometric measurements during the weight maintenance phase (week 14). For FO, there were significant reductions in weight $-1.9 \% \quad(-1.69 \mathrm{~kg}), \quad$ BMI $-1.8 \% \quad\left(-0.6 \mathrm{~kg} / \mathrm{m}^{2}\right)$ ( $P=0.01$ for both), FM $-5 \%(-1.62 \mathrm{~kg}, P=0.03)$, WC $-2.4 \%$ $(-2.29 \mathrm{~cm}, \quad P=0.003)$ and hip circumference $-2.1 \%$ $(-2.38 \mathrm{~cm}, P \leq 0.001)$. For $\mathrm{PB}$, the changes from weight loss to weight maintenance were not significant. A comparison of changes between FO and PB showed no significant differences. For FO, there was a positive, significant correlation between DHA and weight loss $(P=0 \cdot 02)$ and loss of FM $(P=0 \cdot 025)$.

\section{Blood biomarkers}

Weight loss phase. The changes in blood lipids from baseline to the end of weight loss (week 4) are summarised in Table 3. For both FO and $\mathrm{PB}$, there were significant reductions in total cholesterol (TC) $(P<0 \cdot 001$ and $P \leq 0 \cdot 002$, respectively), and LDL-cholesterol (LDL-C) $(P<0.05$ for both), with small, non-significant differences between the two groups. There were positive, significant correlations between weight loss and TC for FO $(P<0.05)$ and PB $(P \leq 0.001)$, and also between weight loss and LDL-C for FO $(P<0.05)$ and $\mathrm{PB}$ $(P \leq 0.001)$. For $\mathrm{FO}$, there were significant reductions in TAG $(P<0.001)$ but not for $\mathrm{PB}$. For both $\mathrm{FO}$ and $\mathrm{PB}$, there were significant reductions in HDL-C $(P<0.01$ for both). There was

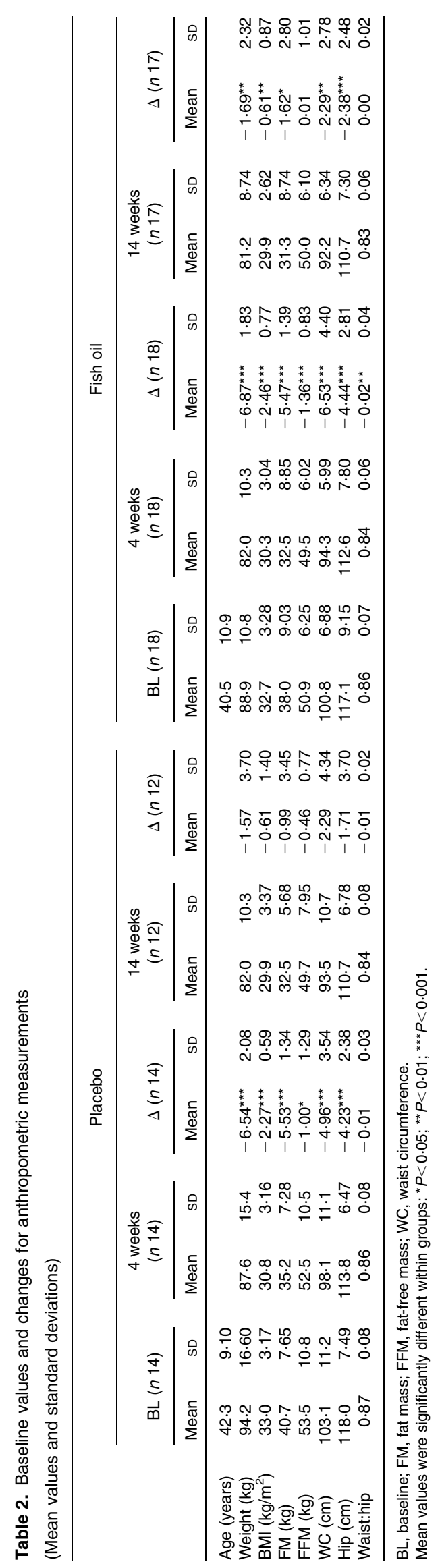


a significant reduction in glucose for FO $(P<0.05)$ but not for $\mathrm{PB}$. The only significant reduction observed in the inflammatory biomarkers was for leptin $(P<0.001$ for $\mathrm{FO}$ and $\mathrm{PB})$ and high-sensitivity IL-6 $(P<0 \cdot 05)$ for PB (Table 3$)$.

Weight maintenance phase. At the end of 10 weeks of weight maintenance (week 14 in the study), there were more changes in blood lipids for both $\mathrm{FO}$ and $\mathrm{PB}$, as shown in Table 3. An increase in TAG and TC was significant for FO and PB $(P<0.05$ for both) and in HDL-C for PB only $(P<0.01)$ (Table 3). HDL-C did increase for FO, but the difference was not significant. There was a significant decrease in levels of high-sensitivity TNF- $\alpha$ and a significant increase in adiponectin levels for FO ( $P \leq 0.05$ for both) (Table 3 ). Other changes in inflammatory biomarkers were non-significant apart from leptin which increased in PB $(P<0 \cdot 01)$ (Table 3$)$. The differences between the two groups were not significant.

\section{Discussion}

The main purpose of the present study was to investigate whether LCn-3PUFA would assist weight loss when administered as a supplement during a weight loss diet. To this end, it was essential that the diet was controlled and participant energy intake was as similar as possible to enable the measurement of the effect of the LCn-3PUFA. By using MR, participants were able to follow a VLED of $3000 \mathrm{~kJ} / \mathrm{d}$ for 4 weeks. Dietary compliance is more favourable with MR than with a conventional reduced energy diet because the prescribed meal content is set; that is, portion sizes are predetermined, food choice is largely removed and so too are the temptations presented when preparing food. MR are also satiating, with the effect lasting for between 3 and $5 \mathrm{~h}$ after consumption $^{(23)}$, thus further facilitating dietary compliance.

Significant reductions in weight, FM, FFM, WC and hip measurements for both groups in this study highlight the efficacy of the VLED, but fail to show a significant weight loss difference from supplementation with LCn-3PUFA, despite the significant increase in plasma concentrations of EPA and DHA. There is conflicting evidence on the effect of LC $n$-3PUFA on weight loss. Some animal studies have shown that supplementation with LC $n$-3PUFA is effective in reducing weight and FM in mice ${ }^{(17)}$ while others have shown no significant effect ${ }^{(24,25)}$. Studies involving human subjects have also reported conflicting results. A small number of severely obese women following a VLED reported a significant weight loss in the group that also consumed LC $n$-3PUFA $(2 \cdot 8 \mathrm{~g} / \mathrm{d} n$-3; EPA:DHA $=2: 1)$ compared to the control $^{(26)}$. A significantly greater weight loss was observed in males but not in females consuming an energy-restricted diet including lean fish $(0.27 \mathrm{~g} / \mathrm{d} n-3 ; 0.05 \mathrm{~g} / \mathrm{d} \mathrm{EPA}+0.21 \mathrm{~g} / \mathrm{d}$ DHA, in the ratio $1: 4)$, or fatty fish $(3.0 \mathrm{~g} / \mathrm{d} n-3 ; 0.77 \mathrm{~g} / \mathrm{d}$ $\mathrm{EPA}+1.37 \mathrm{~g} / \mathrm{d}$ DHA, in the ratio $1: 1 \cdot 8)$ or LCn-3PUFA $(1.42 \mathrm{~g} / \mathrm{d} n-3 ; 0.63 \mathrm{~g} / \mathrm{d} \mathrm{EPA}+0.43 \mathrm{~g} / \mathrm{d}$ DHA, in the ratio $1 \cdot 5: 1)$, compared to the control ${ }^{(14,27)}$. However, no significant differences were observed when participants were given LCn-3 PUFA supplements (1.3 g/d EPA and $2.9 \mathrm{~g} / \mathrm{d}$ DHA, in the ratio $1: 2 \cdot 2)$ in combination with a $\operatorname{VLED}^{(9)}$, or with an energy-controlled diet plus exercise $(3 \mathrm{~g} / \mathrm{d} \mathrm{EPA}+\mathrm{DHA}$, in 
the ratio $5: 1)^{(28)}$, or with energy-controlled but not -restricted diets $(1.1 \mathrm{~g} / \mathrm{d} \text { EPA and } 0.7 \mathrm{~g} / \mathrm{d} \text { DHA, in the ratio } 1.6: 1)^{(29)}$, $(1.8 \mathrm{~g} / \mathrm{d} n-3 ; 1.08 \mathrm{~g} / \mathrm{d}$ EPA and $0.72 \mathrm{~g} / \mathrm{d}$ DHA, in the ratio $1 \cdot 5: 1)^{(30)}$, although there was a significantly greater decrease in FM in the treatment groups compared to the control for these last two studies. A significant weight gain has also been observed in some studies when the diet of cancer patients was supplemented with LC $n-3$ PUFA $^{(31)}$.

To explain the reason for these conflicting results is difficult. It is possible that the energy level of the diet that accompanies supplementation could be a factor. However, in two studies that used a VLED with LC $n$-3 PUFA supplementation, one study reported a significant weight loss compared to the control $^{(26)}$, while the other study reported no significant differences $^{(9)}$. It is also possible that the amount of LC $n$-3PUFA consumed, or the proportion of EPA to DHA could be a contributing factor. Studies with mice have reported that body weight and FM decrease relative to the amount of LCn-3PUFA in the $\operatorname{diet}^{(16)}$, and it has also been shown that LCn-3PUFA prevent weight gain ${ }^{(16,17)}$. Similar findings have been reported in human subjects, with significantly lower levels of LCn-3PUFA found in obese females and/or males compared to healthy-weight individuals ${ }^{(32-34)}$. It would appear that the accumulation of LCn-3PUFA in the body could be an important factor for weight management; so we investigated this possibility in the 10 -week weight maintenance phase.

It is a commonly held belief that slow weight loss is better preserved in the long term ${ }^{(35)}$ and that large amounts of initial weight loss lead to more weight regain ${ }^{(36)}$. However, it has also been reported that a greater initial weight loss is associated with a more significantly sustained weight loss maintenance $^{(35,37)}$. The potential is there for a weight loss rebound after a VLED unless new manageable and effective dietary habits are established. In this study, the VLED was supported with a 10 -week transition period of weight maintenance using the Australian Guide to Healthy Eating to establish healthy eating habits while participants continued to consume the supplements they had been allocated during the weight loss phase. The significant decreases in weight, BMI, FM, WC and hip measurements for FO but not for PB during this phase would appear to support the suggestion that the accumulation of LCn-3PUFA in the body might contribute to weight management. This was further supported by the significant correlations between the level of DHA and reduction in weight, BMI and FM $(P=0.021,0.024$ and 0.025 , respectively) for FO during this phase.

As anticipated, weight loss had a positive influence on blood lipids, which is highlighted by the significant relationships with weight loss and reductions in TC and LDL-C in both the FO and the $\mathrm{PB}$ groups. The reduction in HDL-C levels was similar for both groups. Reductions in levels of TC and LDL-C are associated with clinical benefits, but a reduction in HDL-C is undesirable because of its recognised cardioprotective effects ${ }^{(38)}$. There are conflicting reports on the effect of weight loss on HDL-C, with studies either showing small increases or small decreases or no change at all ${ }^{(39)}$. Overall, it would appear that HDL-C levels decrease with active weight loss and then increase when a stabilised reduced weight is reached ${ }^{(40,41)}$. It has been suggested that the reduction in HDL-C occurs because lipoprotein lipase is reduced during active weight loss, subsequently reducing TAG-rich lipoprotein synthesis which impairs VLDL-C catabolism and the transfer of lipids to HDL-C, thereby reducing HDL-C concentrations. With the stabilisation of weight at the reduced level, lipoprotein lipase increases as does hydrolysis of VLDL-C and the transfer of lipids to HDL-C resumes ${ }^{(40)}$. After the 10 weeks of weight maintenance in our study, HDL-C levels did increase for both groups, but significantly only for the $\mathrm{PB}$ group, reaching a level higher than that at baseline. Levels of TAG, TC and LDL-C also increased, probably as a consequence of resuming the consumption of normal meals with a different fat content. However, they remained lower than the baseline levels.

It has been reported that LC $n$-3PUFA have no effect on TC levels ${ }^{(12,42)}$, although there are differences of opinion on whether LC $n$-3PUFA increase levels of LDL-C $\mathrm{C}^{(42)}$ or have no effect $^{(12)}$. Recent studies ${ }^{(43,44)}$ have shown that LC $n$-3PUFA supplementation results in a small increase in LDL-C; however, it is accompanied by an increase in the size of these particles which are less atherogenic ${ }^{(45)}$. It is well known that LC $n$-3PUFA are effective in lowering TAG levels ${ }^{(46)}$, which supports our findings of a significant reduction in TAG in FO only. During weight loss, there were no significant correlations between blood lipids and EPA or DHA, though there was for glucose which reduced significantly and was

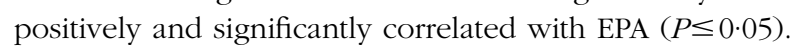

It would appear that weight loss and LC n-3PUFA, either independently or combined, influenced positive changes in the inflammatory markers. While the changes were modest, some of the changes within the groups were significant but, between the groups, they were not. Overall, the study was not adequately powered to determine significance between the groups with such small changes.

Leptin is important for the regulation of food intake, energy expenditure and adiposity ${ }^{(47)}$; and decreasing leptin levels are associated with dietary energy restriction ${ }^{(48)}$ and weight loss ${ }^{(49)}$, while a return to a regular food intake is associated with increased leptin levels ${ }^{(50)}$. Accordingly, in our study, there was a significant decrease in plasma leptin within both groups with dietary energy restriction and weight loss. A return to regular food intake during maintenance saw a significant increase in leptin for PB only, with a very modest increase in leptin for $\mathrm{FO}$, the difference between the two groups almost reaching significance $(P=0.052)$, suggesting a possible influence of LCn-3PUFA on leptin levels in the FO group. A study by Mori et al. ${ }^{(51)}$ reported that a significant reduction in serum leptin was influenced by an interaction between LCn-3PUFA and energy restrictions, but there were no significant effects from LCn-3PUFA or weight loss alone. However, the evidence on the effect of fish oil on plasma leptin levels, in association with changes in adiposity or independent of adipose tissue mass, shows very conflicting results $^{(52)}$.

Reduced levels of CRP, a surrogate marker of CVD risk, are directly related to a reduction in weight loss ${ }^{(53)}$. However, 
in our study, there was a 2-fold greater decrease in CRP levels for $\mathrm{FO}$ during the weight loss phase compared to $\mathrm{PB}$, and $\mathrm{CRP}$ continued to decrease during the maintenance phase for $\mathrm{FO}$ while it increased for $\mathrm{PB}$. This would suggest that the greater decrease in CRP for FO could be attributed to LC $n$-3PUFA. Studies investigating the effect of LCn-3PUFA on CRP have reported conflicting results, with one study finding that LCn-3PUFA intake but not weight loss was associated with a significant reduction in $\mathrm{CRP}^{(54)}$, and the other reporting that LCn-3PUFA had no effect on serum concentrations of $\mathrm{CRP}^{(55)}$. Differences in the size and age of the two groups studied and the length of the intervention could account for the conflicting results. It has also been suggested that such contradictory findings could be attributed to other components in the diet, which might positively affect plasma CRP ${ }^{(56)}$.

Weight loss but not the composition of the diet is reported to be the influence in the reduction of IL- 6 and TNF- $\alpha{ }^{(8)}$. However, a study involving twenty-eight healthy subjects reported that dietary supplementation with fish oil can suppress the production of TNF- $\alpha^{(57)}$. Our present study did not show any positive additional effects of LC $n$-3PUFA on changes to IL- 6 or TNF$\alpha$, which agrees with the findings of a study involving healthy, moderately hypertriacylglycerolaemic subjects where supplementation with $0.85 \mathrm{~g} \mathrm{EPA} / \mathrm{d}$ and $3.4 \mathrm{~g}$ DHA/d over 8 weeks had no effect on the levels of IL- 6 and TNF- $\alpha^{(58)}$. It has been suggested that, as IL- 6 is secreted by adipose tissue, loss of FM with weight loss could contribute to the reduction of IL- $6^{(8)}$. Adiponectin has been reported to increase in response to diet-induced weight loss ${ }^{(59)}$, and LCn-3PUFA ${ }^{(54)}$, although the change in the latter was not significant, possibly because of the small sample size. In our study, weight loss resulted in an increase in adiponectin for PB but decreased for FO, with both not being significant. There was, however, a significant increase in adiponectin levels for FO during maintenance, indicating a possible delayed influence from LC $n$-3PUFA. It has been suggested that a weight loss $>10 \%$ is needed for a significant increase in adiponectin levels to occur ${ }^{(59)}$.

The ratio of plasma 18:1/18:0 is used as a measure of stearoyl-coenzyme A desaturase activity in humans ${ }^{(60)}$ that has been in turn been implicated in weight gain and TAG synthesis. In the present study, the change in the $18: 1 / 18: 0$ ratio was significantly lower in FO compared to the $\mathrm{PB}$ group $(P<0.05)$ (Table 1). The reduction in the 18:1/18:0 ratio in the FO group correlated negatively with change in plasma TAG levels but not with change in BMI or body weight. Considering that TAG levels in plasma correlate with adipose tissue $^{(60)}$, future studies may examine the longer-term effects of fish oil supplementation on body weight and FM.

In summary, it appears that short-term supplementation with LCn-3PUFA did improve the metabolic profile of the participants for the duration of the study, but it did not have any influence on weight loss. There was a significant decrease in FM for the FO group during the maintenance phase but, compared to the PB group, the difference was modest. While it is possible that this difference in FM could be attributed to supplementation with LCn-3PUFA, a larger cohort of subjects would be required to determine the significance with such modest changes. Also, the present study does not rule out the possibility that a deficiency of $n-3$ fatty acids may cause rapid weight gain. The role of $n-3$ fatty acids in weight gain has not been previously examined and is worthy of further examination. The potential benefits of loading the body cells/membranes with $n$ - 3 fatty acids before implementation of the weight loss programme merits further examination.

\section{Study limitation}

A limitation of this study is the small sample size from the reduced completion rate. More studies with a larger sample size and possibly a longer weight stabilisation/maintenance phase are needed to further investigate these findings.

\section{Acknowledgements}

NuMega Ingredients Pty Limited, Australia, supplied the fish oil and Sunola oil capsules. Nestlé Nutrition, Australia, supplied the Optifast ${ }^{\circledR}$ bars and shakes for the MR. Miss Melinda Phang (Nutraceuticals Research Group) provided assistance with the analysis of plasma fatty acids. This research received no specific grant from any funding agency in the public, commercial or not-for-profit sectors. Both authors contributed to the design of the research project. I. A. M. conducted the research, performed the statistical analysis and drafted the manuscript. M. L. G. provided essential materials and contributed to critical review. Both authors read and approved the final manuscript. The authors have no conflicts of interest to declare.

\section{References}

1. Fruhbeck G (2007) Vasoactive factors and inflammatory mediators produced in adipose tissue. In Adipose Tissue and Adipokines in Health and Disease, pp. 63-77 [G Fantuzzi and T Mazzone, editors]. Totowa: Human Press, Inc.

2. NCEP (2002) Third Report of the National Cholesterol Education Program (NCEP) Expert Panel on Detection, Evaluation, and Treatment of High Blood Cholesterol in Adults (Adult Treatment Panel III) final report. Circulation 106, 3143-3421.

3. Weisberg SP, McCann D, Desai M, et al. (2003) Obesity is associated with macrophage accumulation in adipose tissue. J Clin Invest 112, 1796-1808.

4. Blake GJ \& Ridker PM (2001) High sensitivity C-reactive protein for predicting cardiovascular disease: an inflammatory hypothesis. Eur Heart J 22, 349-352.

5. Lau DC, Dhillon B, Yan H, et al. (2005) Adipokines: molecular links between obesity and atheroslcerosis. Am J Physiol Heart Circ Physiol 288, H2031-H2041.

6. Blake GJ \& Ridker PM (2001) Novel clinical markers of vascular wall inflammation. Circ Res 89, 763-771.

7. Nesto RW (2005) Obesity: a major component of the metabolic syndrome. Tex Heart Inst J 32, 387-389.

8. Jellema A, Plat J \& Mensink RP (2004) Weight reduction, but not a moderate intake of fish oil, lowers concentrations of inflammatory markers and PAI-1 antigen in obese men during the fasting and postprandial state. Eur J Clin Invest 34, 766-773.

9. Krebs JD, Browning LM, McLean NK, et al. (2006) Additive benefits of long-chain $n-3$ polyunsaturated fatty acids and weight-loss in the management of cardiovascular disease 
risk in overweight hyperinsulinaemic women. Int $J$ Obes (Lond) 30, 1535-1544.

10. Williamson DF (1997) Intentional weight loss: patterns in the general population and its association with morbidity and mortality. Int J Obes Relat Metab Disord 21, Suppl. 1, S14-S19, discussion S20-S11.

11. Calder PC (2002) Dietary modification of inflammation with lipids. Proc Nutr Soc 61, 345-358.

12. Harris WS (1989) Fish oils and plasma lipid and lipoprotein metabolism in humans: a critical review. J Lipid Res 30, 785-807.

13. Belalcazar LM, Reboussin DM, Haffner SM, et al. (2010) Marine omega-3 fatty acid intake: associations with cardiometabolic risk and response to weight loss intervention in the Look AHEAD (Action for Health in Diabetes) study. Diabetes Care 33, 197-199.

14. Gunnarsdottir I, Tomasson H, Kiely M, et al. (2008) Inclusion of fish or fish oil in weight-loss diets for young adults: effects on blood lipids. Int J Obes (Lond) 32, 1105-1112.

15. Harris WS (1996) n-3 Fatty acids and lipoproteins: comparison of results from human and animal studies. Lipids 31, 243-252.

16. Nakatani T, Kim HJ, Kaburagi Y, et al. (2003) A low fish oil inhibits SREBP-1 proteolytic cascade, while a high-fish-oil feeding decreases SREBP-1 mRNA in mice liver: relationship to anti-obesity. J Lipid Res 44, 369-379.

17. Ruzickova J, Rossmeisl M, Prazak T, et al. (2004) Omega-3 PUFA of marine origin limit diet-induced obesity in mice by reducing cellularity of adipose tissue. Lipids 39, $1177-1185$.

18. Buckley JD \& Howe PR (2009) Anti-obesity effects of longchain omega-3 polyunsaturated fatty acids. Obes Rev 10, 648-659.

19. Blackburn G (1995) Effect of degree of weight loss on health benefits. Obes Res 3, Suppl. 2, 211s-216s.

20. Klein S (2001) Outcome success in obesity. Obes Res 9, Suppl. 4, 354S-358S.

21. Kellet E, Smith AE \& Schmerlaib Y (1998) The Australian Guide to Healthy Eating. Canberra: Australian Government Department of Health and Ageing.

22. Lepage G \& Roy CC (1986) Direct transesterification of all classes of lipids in a one step reaction. J Lipid Res 27, $114-120$.

23. Rothacker DQ \& Watemberg S (2004) Short-term hunger intensity changes following ingestion of a meal replacement bar for weight control. Int J Food Sci Nutr 55, 223-226.

24. Raclot T, Groscolas R, Langin D, et al. (1997) Site-specific regulation of gene expression by $n-3$ polyunsaturated fatty acids in rat white adipose tissues. J Lipid Res 38, 1963-1972.

25. Minami A, Ishimura N, Sakamoto S, et al. (2002) Effect of eicosapentaenoic acid ethyl ester $v$. oleic acid-rich safflower oil on insulin resistance in type 2 diabetic model rats with hypertriacylglycerolaemia. Br J Nutr 87, 157-162.

26. Kunesova M, Braunerova R, Hlavaty P, et al. (2006) The influence of $n-3$ polyunsaturated fatty acids and very low calorie diet during a short-term weight reducing regimen on weight loss and serum fatty acid composition in severely obese women. Physiol Res 55, 63-72.

27. Thorsdottir I, Tomasson H, Gunnarsdottir I, et al. (2007) Randomized trial of weight-loss-diets for young adults varying in fish and fish oil content. Int J Obes (Lond) 31, 1560-1566.

28. DeFina LF, Marcoux LG, Devers SM, et al. (2011) Effects of omega-3 supplementation in combination with diet and exercise on weight loss and body composition. Am J Clin Nutr 93, 455-462.
29. Couet C, Delarue J, Ritz P, et al. (1997) Effect of dietary fish oil on body fat mass and basal fat oxidation in healthy adults. Int J Obes Relat Metab Disord 21, 637-643.

30. Kabir M, Skurnik G, Naour N, et al. (2007) Treatment for 2 mo with $n 3$ polyunsaturated fatty acids reduces adiposity and some atherogenic factors but does not improve insulin sensitivity in women with type 2 diabetes: a randomized controlled study. Am J Clin Nutr 86, 1670-1679.

31. Colomer R, Moreno-Nogueira JM, Garcia-Luna PP, et al. (2007) $N$-3 fatty acids, cancer and cachexia: a systematic review of the literature. BrJ Nutr 97, 823-831.

32. Klein-Platat C, Drai J, Oujaa M, et al. (2005) Plasma fatty acid composition is associated with the metabolic syndrome and low-grade inflammation in overweight adolescents. Am J Clin Nutr 82, 1178-1184.

33. Karlsson M, Marild S, Brandberg J, et al. (2006) Serum phospholipid fatty acids, adipose tissue, and metabolic markers in obese adolescents. Obesity (Silver Spring) 14, 1931-1939.

34. Micallef M, Munro I, Phang M, et al. (2009) Plasma $n$-3 polyunsaturated fatty acids are negatively associated with obesity. Br J Nutr 102, 1370-1374.

35. Astrup A \& Rossner S (2000) Lessons from obesity management programmes: greater initial weight loss improves long-term maintenance. Obes Rev 1, 17-19.

36. McGuire MT, Wing RR, Klem ML, et al. (1999) What predicts weight regain in a group of successful weight losers? J Consult Clin Psychol 67, 177-185.

37. Anderson JW, Konz EC, Frederich RC, et al. (2001) Long-term weight-loss maintenance: a meta-analysis of US studies. $A m \mathrm{~J}$ Clin Nutr 74, 579-584.

38. Boden WE (2000) High-density lipoprotein cholesterol as an independent risk factor in cardiovascular disease: assessing the data from Framingham to the Veterans Affairs HighDensity Lipoprotein Intervention Trial. Am J Cardiol 86, 19L-22L

39. Poobalan A, Aucott L, Smith WC, et al. (2004) Effects of weight loss in overweight/obese individuals and long-term lipid outcomes - a systematic review. Obes Rev 5, 43-50.

40. Dattilo AM \& Kris-Etherton PM (1992) Effects of weight reduction on blood lipids and lipoproteins: a meta-analysis. Am J Clin Nutr 56, 320-328.

41. Ditschuneit HH, Flechtner-Mors M, Johnson TD, et al. (1999) Metabolic and weight-loss effects of a long-term dietary intervention in obese patients. Am J Clin Nutr 69, 198-204.

42. Balk EM, Lichtenstein AH, Chung M, et al. (2006) Effects of omega-3 fatty acids on serum markers of cardiovascular disease risk: a systematic review. Atherosclerosis 189, 19-30.

43. Micallef MA \& Garg ML (2008) The lipid-lowering effects of phytosterols and ( $n-3)$ polyunsaturated fatty acids are synergistic and complementary in hyperlipidemic men and women. J Nutr 138, 1086-1090.

44. Micallef MA \& Garg ML (2009) Anti-inflammatory and cardioprotective effects of $n-3$ polyunsaturated fatty acids and plant sterols in hyperlipidemic individuals. Atherosclerosis 204, 476-482.

45. Connor WE (1968) Dietry sterols: their relationship to atherosclerosis. J Am Diet Assoc 52, 202-208.

46. Harris WS \& Bulchandani D (2006) Why do omega-3 fatty acids lower serum triglycerides? Curr Opin Lipidol $\mathbf{1 7}$ 387-393.

47. Anubhuti \& Arora S (2008) Leptin and its metabolic interactions: an update. Diabetes Obes Metab 10, 973-993.

48. Boden G, Chen X, Mozzoli M, et al. (1996) Effect of fasting on serum leptin in normal human subjects. J Clin Endocrinol Metab 81, 3419-3423. 
49. Maffei M, Halaas J, Ravussin E, et al. (1995) Leptin levels in human and rodent: measurement of plasma leptin and ob RNA in obese and weight-reduced subjects. Nat Med 1, $1155-1161$.

50. Kolaczynski JW, Considine RV, Ohannesian J, et al. (1996) Responses of leptin to short-term fasting and refeeding in humans: a link with ketogenesis but not ketones themselves. Diabetes 45, 1511-1515.

51. Mori TA, Burke V, Puddey IB, et al. (2004) Effect of fish diets and weight loss on serum leptin concentration in overweight, treated-hypertensive subjects. J Hypertens 22, 1983-1990.

52. Puglisi MJ, Hasty AH \& Saraswathi V (2011) The role of adipose tissue in mediating the beneficial effects of dietary fish oil. J Nutr Biochem 22, 101-108.

53. Selvin E, Paynter NP \& Erlinger TP (2007) The effect of weight loss on C-reactive protein: a systematic review. Arch Intern Med 167, 31-39.

54. Tsitouras PD, Gucciardo F, Salbe AD, et al. (2008) High omega-3 fat intake improves insulin sensitivity and reduces CRP and IL6, but does not affect other endocrine axes in healthy older adults. Horm Metab Res 40, 199-205.

55. Madsen T, Christensen JH, Blom M, et al. (2003) The effect of dietary $n-3$ fatty acids on serum concentrations of
C-reactive protein: a dose-response study. Br J Nutr 89 , 517-522.

56. Puglisi MJ \& Fernandez ML (2008) Modulation of C-reactive protein, tumor necrosis factor-alpha, and adiponectin by diet, exercise, and weight loss. J Nutr 138, 2293-2296.

57. Caughey GE, Mantzioris E, Gibson RA, et al. (1996) The effect on human tumor necrosis factor alpha and interleukin 1 beta production of diets enriched in $n-3$ fatty acids from vegetable oil or fish oil. Am J Clin Nutr 63, 116-122.

58. Skulas-Ray AC, Kris-Etherton PM, Harris WS, et al. (2011) Dose-response effects of omega-3 fatty acids on triglycerides, inflammation, and endothelial function in healthy persons with moderate hypertriglyceridemia. Am J Clin Nutr 93, 243-252.

59. Madsen EL, Rissanen A, Bruun JM, et al. (2008) Weight loss larger than $10 \%$ is needed for general improvement of levels of circulating adiponectin and markers of inflammation in obese subjects: a 3-year weight loss study. Eur J Endocrinol 158, 179-187.

60. Attie AD, Krauss RM, Gray-Keller MP, et al. (2002) Relationship between stearoyl-CoA desaturase activity and plasma triglycerides in human and mouse hypertriglyceridemia. J Lipid Res 43, 1899-1907. 